\title{
A Framework for Deterministic Intention Specifications of an Agent toward an Incomplete Declared Intention
}

\author{
Dussadee Praserttitipong and Peraphon Sophatsathit \\ Advanced Virtual and Intelligent Computing (AVIC) Center, \\ Department of Mathematics, Faculty of Science, \\ Chulalongkorn University, Bangkok, 10330, Thailand. \\ Email: dussadee@ science.cmu.ac.th, Peraphon.S@Chula.ac.th
}

\begin{abstract}
An agent typically operates in dynamic and unpredictable environments. The ability of an agent to adapt according to changes in the environments is important in enabling dynamic and reactive behaviors. This paper proposes a behavioral model for agent's deterministic intention specifications that enable intelligent adaptation capabilities of the agent to accommodate the user's purpose. Additional scalable capability on finite domain of knowledge is applied toward the specifications. This permits the agent to be potent enough to accomplish its tasks over either a complete or incomplete knowledge stated in a given intention specifcation. Accuracy of the result is measured by precision and recall as to how well the proposed model performs.
\end{abstract}

\section{Introduction}

An intelligent software agent is a type of software agent having built-in intelligent behavior to handle the tasks in accordance with a priori knowledge. The agent usually operates in dynamic and uncertain environments whose information about the world is not all known or adequately characterized in advance. One approach to enable such intelligent adaptation capability utilizes service discovery.

In this approach, the agent contacts a middle agent, called service broker, to determine the most suitable service according to the agent's intention specification. Upon receiving the intention specification, the service broker performs a sequence of service discovery using matchmaking procedures. This process provides recommendations for suitable services based upon service advertisement. In so doing, the agent will be able to accomplish its intention in the dynamic and uncertain environments.

The objective of this paper is to introduce a deterministic intention specification focusing on behavioral model under complete and incomplete agent's intentions. A deterministic matchmaking process is provided to ensure that the user's purpose is responded. The rest of this paper is organized as follows. Section 2 reviews some related works. The proposed approach is introduced in Section 3. An empirical experiment and its evaluation are presented in Section 4 and 5, respectively. Section 6 concludes the paper with some final thoughts.

\section{Related Works}

Current service discovery approaches shift toward the paradigm known as table-based service discovery [10], running along side with current standard service discovery technologies. The advertisement of services from both inhouse libraries and third-party on-line repositories over the network are discovered by considering a fixed number of attributes describing functional properties of the service. These literatures suggest that there have been growing interests in focusing on functional specification rather than on behavioral specification.

However, some inherent problems concerning with a table-based service discovery persist. Because the matchmaking process relies on keywords matching, some services might be overlooked. There have been intensive researches emphasizing on addressing this problem using semantic concepts [5, 6], ontology, and context-awareness [2, 7, 8]. These are considered as successful principles for search quality improvement. However, these studies have merely emphasized on modeling functional specifications with less attention paid on behavioral specifications. The behavioral specification is normally described in natural language. Thus, matchmaking process performing on the behavioral specification operates on the principle of information retrieval [5]. We refer to these investigations as conventional nondeterministic specifications. It remains unclear whether the details about how the actual service works in each exe- 
cution step covering the user's intention. Such loose or lack of concern on service behavior often leads to nondeterministic agent execution.

It would seem, therefore, that further investigations are needed in order to arrive at some forms of deterministic capability given a conventional intention specification model. We will look into design time deterministic service discovery. Special attention will be paid on deductive service discovery $[3,11]$ which aims at expressing the service behavior and semantics using formal specification and logic modeling, respectively. These specifications are formally represented [9] so as to facilitate a more practical matchmaking process.

\section{Proposed Approach}

There are two underlying processes in the proposed service discovery mechanism, i.e., service specification advertising and deterministic behavioral elicitation algorithm. The former is a service specification advertisement focusing on how to advertise the service, while the latter is a deterministic behavioral elicitation algorithm that focuses on how to select the most suitable service. Once service specification advertisement is established, the algorithm will aid in matching suitable service to behaviorally adapt the agent's intention specifications. Performance measure of service matching and adaptation are assessed by precision and recall. Details of the proposed approach will be elucidated in the sections that follow.

\subsection{Fundamentals}

The emphasis of this research rests on the notion of conventional nondeterministic specification. There are two crucial points that need to be addressed.

1. In order to overcome an infinite set of possible actions, all elements in the set of possible actions are reduced to user's attitude toward a particular decision criterion. The definition of attitude is defined in [4] as follows:

Attitude can be defined as a predisposition that is learned in order to respond in a consistent way, either in favorable or unfavorable manner toward a specific object.

Hence, the qualitative judgment of each condition statement is formalized by a bipolar attitude scale as favorable and unfavorable classes toward the agent's decision (that is formulated from the users attitude) acting on behalf of the user. In so doing, possible actions stating in this proposed behavioral specification model can be narrated in discrete or finite terms. Obviously, using discrete/finite terms is more practical than descriptive/infinite terms. The necessary preamble assumption of this proposed approach stipulates that an infinite set of possible actions could be reduced to a corresponding finite set of values or categories.

2. The problem of indescribable situation brings about exceeded capabilities of a complete service advertisement comparing with an incomplete agent intention specification. This is due to some situations where results could fall outside the boundaries of the agent intention specification. These situations, in many cases, tend to be indescribable situations for the agent. In addition, conventional deterministic matching is performed over fixed number of attributes, or white box specification in this literature. Any unspecified or new attributes are considered indescribable which, from theoretical standpoint, must be extended (scaling) to accommodate the extraneous matching requirements. Unfortunately, scalable capability is unlikely to carry out. Such a limitation imposes difficulties in dealing with those indescribable situations.

In this paper, an ordinary deterministic white box specification, which can be either a complete or an incomplete specification, is normalized to a proposed deterministic behavioral specification. The proposed specification, or a deterministic gray box specification, is originated from a machine learning process, hereafter referred to as a normalized deterministic behavior specification. Learning parameters characterizing a service behavior are obtained from neural network techniques. These parameters are embedded as the attribute values of the proposed specification.

Before any matchmaking commences, complete deterministic white box specifications with new or unknown decision making situations are inducted from the learning parameters. These forthcoming specifications are called scaled deterministic white box specifications. Further denotation of the agent intention specification becomes $\beta$ and the service advertisement is referred to as $\pi$. Both scaled$\beta$ and scaled- $\pi$ are arranged in matrix format of unequal size. These matrices are transformed by the proposed algorithm to equal-sized matrices. Consequently, the indescribable problems become scaled intention specification. Four essential definitions are established to facilitate subsequent derivation as follows:

Definition 1 A white box deterministic intention specification

Let $\mathcal{I}^{W B}$ denote a white box deterministic intention specification of either $\beta$ or $\pi$. This specification is defined as $(\delta, \Omega)$, where $\delta$ is an $\alpha \times \mathcal{N}$ matrix. $\mathcal{N}$ is the number of common concerning attribute values. If the $j^{t h}$ attribute value represents the concerning attribute of the $i^{\text {th }}$ condition, 
$\delta(i, j)$ is set to 1 . Otherwise, $\delta(i, j)$ is assigned with 0 . Similarly, for any $i^{\text {th }}$ row in an $\alpha \times 1$ matrix $\Omega, \Omega(i)$ represents a class of actions (the $i^{t h}$ row of $\Omega$ ) which are relevant to a condition statement $\delta(i) . \Omega(i)$ is set to 1 if $\delta(i)$ is FV Class, otherwise $\Omega(i)$ is 0 .

In case of a complete deterministic white box specification, all possible situations must be specified or assessed with their relevant actions. Thus, the variable $\alpha$ is equal to the number of all possible situations $\mathcal{M}$. By contrast, an incomplete deterministic white box specification whose partial situations are specified with their relevant actions, the variable $\alpha$ is equal to the number of declared situations.

\section{Definition 2 A deterministic gray box specification}

Let $\mathcal{I}^{G B}$ denote a deterministic gray box specification of either $\beta$ or $\pi$. This specification comprises a 2-tuple defined as $(\mathcal{W}, b)$, where $\mathcal{W}$ represents an adjusted learning weight matrix and $b$ represents an adjusted learning bias matrix.

For an instance of $\mathcal{I}^{G B}$, say $a$, the general notation is $\mathcal{I}_{a}$ (rather than $\mathcal{I}_{a}^{G B}$ for brevity). Thus, $I_{\beta}$ and $I_{\pi}$ mean deterministic gray box specifications of $\beta$ and $\pi$, respectively.

\section{Definition 3 A compatible degree}

A compatibility degree of reusing a $\mathcal{I}_{b}$ in place of $\mathcal{I}_{a}$, hereafter referred to as $\Theta^{\mathcal{I}_{a}, \mathcal{I}_{b}}$, can be calculated as follows:

$$
\Theta^{\mathcal{I}_{a}, \mathcal{I}_{b}}=\frac{\sum_{i=1}^{\alpha} \Theta_{i}^{\mathcal{I}_{a}, \mathcal{I}_{b}}}{\alpha} \times 100
$$

where $\alpha$ denotes the number of the describable cases which can be assessed by a minimum value between $\alpha^{\mathcal{I}_{a}}$ and $\alpha^{\mathcal{I}_{b}}$. $\Theta^{\mathcal{I}_{a}, \mathcal{I}_{b}}$ is the percentage of compatible degree between $\mathcal{I}_{a}$ and $\mathcal{I}_{b}$ (how many elements of $\mathcal{I}_{a}$ match/similar to $\mathcal{I}_{b}$ ). The compatibility value for the $i^{t h}$ conditional statement, $\Theta_{i}^{\mathcal{I}_{a}, \mathcal{I}_{b}}$, can be expressed as follows:

$$
\Theta_{i}^{\mathcal{I}_{a}, \mathcal{I}_{b}}= \begin{cases}1 & \text { if } \mathcal{I}_{a} \cdot \Omega(i)=\mathcal{I}_{b} \cdot \Omega(i) \\ 0 & \text { otherwise }\end{cases}
$$

where $\mathcal{I}_{a} \cdot \Omega(i)=\mathcal{I}_{b} \cdot \Omega(i)$ if either $\mathcal{I}_{a} \cdot \Omega(i) \in$ $F V$ and $\mathcal{I}_{b} \cdot \Omega(i) \in F V$ or $\mathcal{I}_{a} . \Omega(i) \in$ $U F V$ and $\mathcal{I}_{b} . \Omega(i) \in U F V$, and $\Theta_{i}^{\mathcal{I}_{a}, \mathcal{I}_{b}} \in[0:$ $1]$.

\section{Definition 4 A resemblance degree}

A resemblance degree, $\Gamma^{\mathcal{I}_{a}, \mathcal{I}_{b}}$, is the ability for preserving principal characteristics of $\mathcal{I}_{a}$ in comparison with $\mathcal{I}_{b}$. This degree can be calculated as follows:

$$
\Gamma^{\mathcal{I}_{a}, \mathcal{I}_{b}}=1 /\left[1+\nabla_{\mathcal{E}}^{\mathcal{I}_{a}, \mathcal{I}_{b}}\right]
$$

where $\nabla_{\mathcal{E}}^{\mathcal{I}_{a}, \mathcal{I}_{b}}$ is a discrepancy degree. The discrepancy degree is assessed from the difference between the capabilities stated in $\mathcal{I}_{a}$ and $\mathcal{I}_{b}$ which can be determined by the following equation

$$
\nabla_{\mathcal{E}}^{\mathcal{I}_{a}, \mathcal{I}_{b}}=\sum_{i=1}^{n}\left[e^{\left[\mathcal{E}^{\mathcal{I}_{a}}(j)-\mathcal{E}^{\mathcal{I}_{b}}(j)\right]} \times\left|\mathcal{P}^{\mathcal{I}_{a}}(j)-\mathcal{P}^{\mathcal{I}_{b}}(j)\right|\right] \text { (4) }
$$

where

1. $\mathcal{E}^{\mathcal{I}_{a}}(j)$ and $\mathcal{E}^{\mathcal{I}_{b}}(j)$ are the entropy of $\mathcal{I}_{a}$ and $\mathcal{I}_{b}$ over a concerning attribute value $j^{\text {th }}$. $\mathcal{E}(j)$ of either $\mathcal{I}_{a}, \mathcal{I}_{b}$ is given by

$$
\begin{aligned}
\mathcal{E}(j)= & -\left(\left[\mathcal{P}(j) \times \log _{2} \mathcal{P}(j)\right]\right. \\
& \left.+\left[(1-\mathcal{P}(j)) \times \log _{2}(1-\mathcal{P}(j))\right]\right)
\end{aligned}
$$

2. $\mathcal{P}^{\mathcal{I}_{a}}(j)$ and $\mathcal{P}^{\mathcal{I}_{b}}(j)$ are the probability of $\mathcal{I}_{a}$ and $\mathcal{I}_{b}$ over a concerning attribute value $j^{\text {th }}$ toward the $F V$ class. $\mathcal{P}(j)$ of either $\mathcal{I}_{a}, \mathcal{I}_{b}$ becomes

$$
\mathcal{P}(j)=\frac{\text { number of } \mathcal{A}(j) \text { belonging to } F V \text { class }}{\text { number of occurrences } \mathcal{A}(j)}
$$

\subsection{A Deterministic Behavioral Elaborating Algo- rithm}

Back-propagation learning algorithm [1] is selected as a behavioral elaborating algorithm, using multilayer feedforward network with two-layers $\log$ - sigmoid transfer function. The input vector, $\delta$, represents the declared situations in matrix form operating on three neurons. The output layer utilizes one neuron. The hidden layer, encompassing a log-sigmoid transfer function, employs one neuron. A training function based on conjugate gradient back-propagation with Fletcher-Reeves updates is also embedded in the hidden layer.

Let $\overline{\mathcal{I}}, \hat{\mathcal{I}}$, and $\mathcal{I}^{\prime}$ denote the ordinary deterministic white box specification, the normalized deterministic gray box behavior specification, and the scaled intention specification, respectively. $\overline{\mathcal{I}}$ and $\mathcal{I}^{\prime}$ are structured in the form of $\mathcal{I}^{W B}$, whereas $\hat{\mathcal{I}}$ is arranged in $\mathcal{I}^{G B}$. We denote $\Theta^{\text {threshold }}$, $\nabla_{\mathcal{E}}^{\text {threshold }}$ and maxIter as the accuracy of normalization process, acceptable value of a discrepancy degree, and the maximum iteration for performing a normalization process accordingly. The default values of $\Theta^{\text {threshold }}, \nabla_{\mathcal{E}}^{\text {threshold }}$ and maxIter are set to $90 \%, 0.1$ and 20, respectively. 
A pivotal process of this algorithm is $\overline{\mathcal{I}}-\hat{\mathcal{I}}$ normalization to determine $\hat{\mathcal{I}}$ whether $\hat{\mathcal{I}}$ is, in general, approximately equal or close to $\overline{\mathcal{I}}$. Thus, the results of this step yield components of temporary $\hat{\mathcal{I}}$, namely, 1) $\mathcal{W}^{1}$ a weight matrix of the input $\left(1^{\text {st }}\right)$ layer represented by an $S \times n$ matrix, 2) $\mathcal{W}^{2}$ a weight matrix of the hidden $\left(2^{\text {nd }}\right)$ layer represented by a $1 \times S$ matrix, 3) $b^{1}$ a bias matrix of the input $\left(1^{s t}\right)$ layer represented by an $S \times 1$ matrix, and 4) $b^{2}$ a bias matrix of the hidden $\left(2^{n d}\right)$ layer represented by a $1 \times 1$ matrix, where $S$ is the number of learning neurons. The default value of $S$ is set to $\mathcal{N}$.

\section{Algorithm 1 A deterministic behavior elaborating algo- rithm}

1. Create $\overline{\mathcal{I}}$ consisting matrices $\overline{\mathcal{I}} . \delta$ and $\overline{\mathcal{I}} . \Omega$ from an ordinary rule-based specification, which is a deterministic white box intention specification.

2. Create a complete decision making situation matrix, $\delta^{c}$

\section{REPEAT}

(a) Normalize $\overline{\mathcal{I}}$ to $\hat{\mathcal{I}}$ by creating a 2-layers feed forward network with log-sigmoid transfer function.

(b) Compute a compatible degree $\Theta^{\overline{\mathcal{I}}, \hat{\mathcal{I}}}$ of reusing $\hat{\mathcal{I}}$ in place of $\overline{\mathcal{I}}$ from Equation 1.

(c) Scale up $\hat{\mathcal{I}}$ to $\mathcal{I}^{\prime}$ according to $\delta^{c}$ with $\log$-sigmoid function.

(d) Calculate a discrepancy degree $\nabla_{\mathcal{E}}^{\overline{\mathcal{I}}, \mathcal{I}^{\prime}}$ of $\overline{\mathcal{I}}$ and $\mathcal{I}^{\prime}$ from Equation 4.

(e) $\mathbf{I F}[$ iteration $=1] \mathbf{O R}\left[\Theta^{\overline{\mathcal{I}}, \hat{\mathcal{I}}}<\Theta^{\text {min }}\right] \mathbf{O R}$ $\left[\left(\Theta^{\overline{\mathcal{I}}, \hat{\mathcal{I}}}=\Theta^{\min }\right) \mathbf{A N D}\left(\nabla_{\mathcal{E}}^{\overline{\mathcal{I}}, \mathcal{I}^{\prime}}<\nabla_{\mathcal{E}}^{\min }\right)\right]$
i. $\Theta^{\min }=\Theta^{\overline{\mathcal{I}}, \hat{\mathcal{I}}}$
ii. $\nabla_{\mathcal{E}}^{\min }=\nabla_{\mathcal{E}}^{\overline{\mathcal{I}}, \mathcal{I}^{\prime}}$
iii. Set output $\hat{\mathcal{I}}=\hat{\mathcal{I}}$

\section{ENDIF}

(f) Increase the value of iteration by 1

$$
\begin{aligned}
\text { UNTIL } & {\left[\left(\Theta^{\overline{\mathcal{I}}, \hat{\mathcal{I}}}<\Theta^{\text {threshold }}\right) \mathbf{A N D}\left(\nabla_{\mathcal{E}}^{\overline{\mathcal{I}}, \mathcal{I}^{\prime}}<\nabla_{\mathcal{E}}^{\min }\right)\right] } \\
& \mathbf{O R}[\text { iteration }>\text { maxIter }]
\end{aligned}
$$

According to the process specified in Algorithm 1, these followings conditions must hold true:

1. $\Theta^{\overline{\mathcal{I}}, \hat{\mathcal{I}}}$ must be equal or close to $100 \%$, i.e., $\overline{\mathcal{I}} . \Omega=\hat{\mathcal{I}} . \Omega$, that the decision making of $\hat{\mathcal{I}}$ according to the information specified in $\overline{\mathcal{I}}$ must be equivalent.

2. $\nabla_{\mathcal{E}}^{\hat{\mathcal{I}}, \mathcal{I}^{\prime}}$ must be equal or close to 0 , i.e., $\nabla_{\mathcal{E}} \approx \epsilon$, where $\epsilon$ approaches to zero. The entropy of the original specification $\overline{\mathcal{I}}$ and the assessment of $\mathcal{I}^{\prime}$ must be almost equal.

\subsection{Matchmaking Process}

The matchmaking process is a process of finding the most relevant service in comparison with $\beta$. The process is described in Algorithm 2.

\section{Algorithm 2 A matchmaking algorithm}

1. Create a complete decision making situation matrix, $\delta^{c}$

2. Determine the value of both $\hat{\beta}$ and $\hat{\pi}$ for all possible situations obtained from $\delta^{c}$ with log-sigmoid transfer function.

3. Calculate the compatible degree of $\hat{\pi}$ according to $\hat{\beta}$, $\Theta^{\hat{\beta}, \hat{\pi}}$, in accordance with Equation 1.

4. Find a set of candidate services ( $\Pi$ ) that have the compatible degree within $\sigma$, where $\sigma$ is an acceptable compatible degree of reusing $\pi$ in place of $\beta$.

5. Find the most proper service that has the highest compatible degree. The result of this step is the most proper service, $\tau$. This service is the service having the maximum value of the $\Theta^{\hat{\beta}, \hat{\pi}}$

$$
\left.\tau: \tau \subseteq \tilde{\tau}, \tau=\left\{\tau \mid \forall(\tau), \text { where } \Gamma(\beta, \tau)=\max \left(\Gamma^{\beta, \tilde{\tau}}\right)\right)\right\}
$$

Hence, $\tau$ is the selected service having the smallest discrepancy between $\beta$ and $\pi$.

Consequently, $\sigma$ is used as an indicator to measure user's satisfaction. The candidate services are the services that have a compatible degree greater than an acceptable compatibility value specified by the user.

\section{Experiments}

There were three fundamental approaches conducted in the experiment.

1. $\mathcal{B r o k e r}^{N D}$ performs service validation algorithm on a table-based specification approach which is a nondeterministic behavioral specifications approach.

2. B roker $^{W B}$ performs matchmaking algorithm based on $\mathcal{I}^{W B}$ which is a deterministic behavioral specification approach.

3. Broker $^{G B}$ performs service validation algorithm based on $\mathcal{I}^{G B}$ which is a deterministic behavioral specification approach.

The test cases employed in this paper were generated based on discrete data that were uniformly distributed, i.e., data were evenly drawn from $\beta$ and $\pi$. Two scenarios were considered, namely, equivalent matchmaking and inequivalent 
matchmaking. The former was exercised in case of $\alpha^{\beta}$ and $\alpha^{\pi}$ were equal, whereas the latter took place otherwise. In latter case, scaled deterministic white box specification was performed.

There were 100 test cases of equivalent match performed. Broker $^{W B}$ was then employed to derive a benchmark result, whereby the accuracy of the proposed model could be obtained from the difference between this benchmark result and result obtained from Broker $^{G B}$.

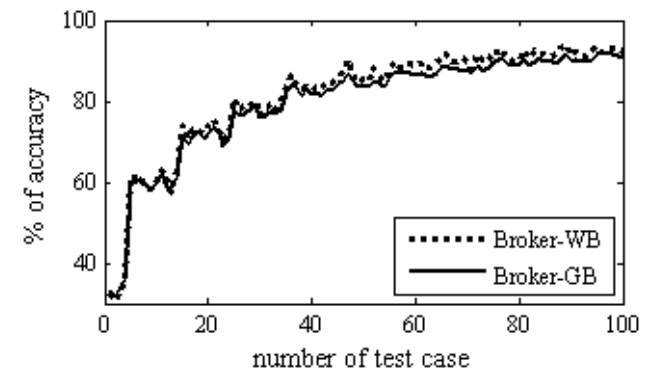

\section{Figure 1. The degree of accuracy of the equiv- alent match}

The value of $\overline{\mathcal{I}}, \hat{\mathcal{I}}$ and $\mathcal{I}^{\prime}$ were applied to determine $\Theta^{\overline{\mathcal{I}}, \hat{\mathcal{I}}}, \Theta^{\hat{\mathcal{I}}, \mathcal{I}^{\prime}}, \nabla_{\mathcal{E}}^{\overline{\mathcal{I}}, \hat{\mathcal{I}}}$, and $\nabla_{\mathcal{E}}^{\overline{\mathcal{I}}, \mathcal{I}^{\prime}}$ based on Algorithm 1. Figure 1 depicts compatibility results from $\mathcal{B}$ roker ${ }^{W B}$ and Broker $^{G B}$, represented by dotted-line and solid-line, respectively. The average and standard deviation differences are $1.62 \%$ and $2.20 \%$. Generally, the lower difference value reflects the higher accuracy degree achieved.

For equivalent matchmaking, similar procedures were carried out based on Algorithm 2. which yielded the accuracy at $98.32 \%$. Inequivalent match, on the other hand, measured the incompute agent intention specification. The size of conditional statement in $\pi \mathrm{s}$ was equal to $\mathcal{M}$, whereas $\beta$ s varied between $10-30 \%$ of $\mathcal{M}$. The assumption of this experiment was that all common attributes has to be stated as concerning attributes of $\beta$ s with uniform probability. In other words, all common attributes had to relate to one or more conditions specified in each test case of $\beta$.

Compatibility results obtained from $\mathcal{B r o k e r}^{W B}$ and Broker $^{G B}$ are compared. The compatible degrees between Broker $^{W B}$ and $\mathcal{B}$ roker ${ }^{G B}$ are $55.34 \%, 98.21 \%$, respectively. The corresponding standard deviation are $14.66 \%$ and $1.79 \%$, respectively. As can be seen from Figure 2, the results achieved by $\mathcal{B}$ roker ${ }^{W B}$ are two times lower than those from Broker $^{G B}$.

\section{Evaluation of the results}

Assessments for quality of matchmaking algorithm were carried out by means of recall and precision measurements.

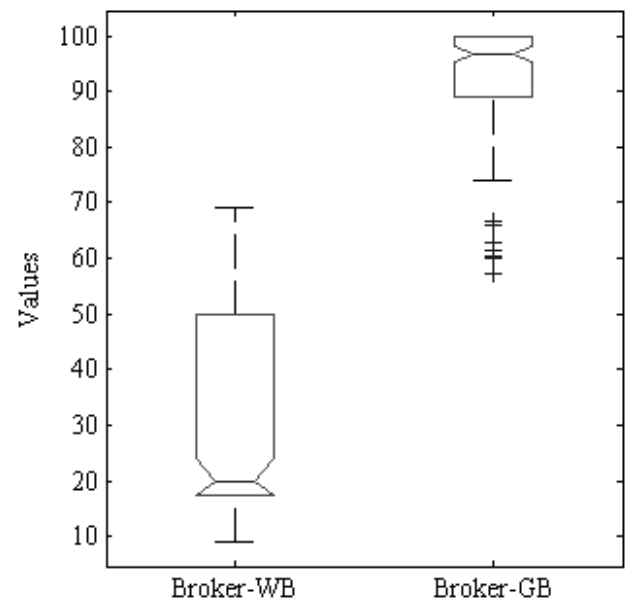

Figure 2. Box plots illustrating the results from the inequivalent match

The experiment was conducted on the blueprints over three groups of data, namely, complete basis intention $\mathcal{G}^{0}$, complete synthetic intention $\mathcal{G}^{1}$, and incomplete basis intention $\mathcal{G}^{2}$. The target $\mathcal{G}^{2}$ symbolized the required $\beta$ s attempting to match with $\mathcal{G}^{0}$ and $\mathcal{G}^{1}$. Due to unequal size of the three groups, in particular $\mathcal{G}^{2}$, scaling (Algorithm 1) was performed. Subsequent matchmaking (Algorithm 2) was carried out to measure $\beta(t)$ of $\mathcal{G}^{2}$ against the $i^{t h}$ service of $\mathcal{G}^{0}$ and $\mathcal{G}^{1}$ through $\mathcal{B}$ roker ${ }^{N D}, \mathcal{B r o k e r}^{W B}$ and $\mathcal{B}_{\text {roker }}{ }^{G B}$. Match measure process went through each element via $\Pi(t)$ as $\Pi(t)=\left\{\pi^{0}(t), \pi^{1}(t), \pi^{2}(t)\right\}$, where $\pi^{0}(t), \pi^{1}(t)$ and $\pi^{2}(t)$ were $t^{t h}$ elements of group $\mathcal{G}^{0}, \mathcal{G}^{1}$ and $\mathcal{G}^{2}$, respectively. Comparative results $\mathcal{B}$ roker ${ }^{N D}$ (nondeterministic match), $\mathcal{B}$ roker ${ }^{W B}$ (conventional deterministic match), and Broker $^{G B}$ (scaled deterministic match) are illustrated in Table 1. As matchmaking process was executing, precision and recall statistics were collected from each Broker mandated by $\Pi$.

Table 1. Instances of results from all Brokers

\begin{tabular}{|l|l|l|l|}
\hline Measurement & $\mathcal{B}$ roker $^{N D}$ & Broker $^{W B}$ & Broker $^{\text {GB }}$ \\
\hline $\begin{array}{l}\text { (1) Set of total relevant } \\
\text { services }\end{array}$ & $\mathcal{G}^{0}(1), \mathcal{G}^{2}(1)$ & $\mathcal{G}^{0}(1), \mathcal{G}^{2}(1)$ & $\mathcal{G}^{0}(1), \mathcal{G}^{2}(1)$ \\
\hline $\begin{array}{l}\text { (2) Set of total services }(\tau) \\
\text { retrieved }\end{array}$ & $\begin{array}{l}\mathcal{G}^{0}(1), \mathcal{G}^{2}(1), \\
\mathcal{G}^{1}(1)\end{array}$ & $\mathcal{G}^{0}(1)$ & $\mathcal{G}^{0}(1), \mathcal{G}^{2}(1)$ \\
\hline $\begin{array}{l}\text { (3) Set of total relevant } \\
\text { services retrieved }\end{array}$ & $\mathcal{G}^{0}(1), \mathcal{G}^{2}(1)$ & $\mathcal{G}^{0}(1)$ & $\mathcal{G}^{0}(1), \mathcal{G}^{2}(1)$ \\
\hline (4) Precision & 0.67 & 1.00 & 1.00 \\
\hline (5) Recall & 1.00 & 0.5 & 1.00 \\
\hline
\end{tabular}

As shown in Table 1, set $\tau$ s obtained from $\mathcal{B}$ roker ${ }^{N D}$ can be regarded as the superset of total relevant service set. 
This evidence shows that some irrelevant services are retrieved in the matchmaking process of $\mathcal{B}$ roker ${ }^{N D}$. Because Broker $^{N D}$ performs a service matchmaking algorithm on a nondeterministic behavioral specification, the behavioral similarity is overlooked.

On the other hand, the same set $\tau$ s obtained from Broker $^{W B}$ can also be regarded as the subset of overall relevant service set. This evidence indicates that some relevant services are unnoticed by $\mathcal{B}$ roker ${ }^{W B}$. This is due to scalability limitation of $\mathcal{B r o k e r}^{W B}$. Table 2 presents

\section{Table 2. Precision and recall}

\begin{tabular}{|l||l|l|l|}
\hline Measurement & Broker $^{N D}$ & Broker $^{W B}$ & Broker $^{G B}$ \\
\hline Precision & 0.67 & 1.00 & 1.00 \\
\hline Recall & 1.00 & 0.50 & 0.99 \\
\hline
\end{tabular}

the results of precision and recall attained from $\mathcal{B}$ roker ${ }^{N D}$, Broker $^{W B}$ and Broker $^{G B}$. As can be seen, precision acquired from $\mathcal{B r o k e r}^{N D}$ is the lowest comparing with other brokers. A different scenario is resulted from recall measure from $\mathcal{B r o k e r}^{W B}$.

Based on the empirical results of $\mathcal{B r o k e r}^{N D}$ and Broker $^{W B}$, representing service functions with loosely or lack of concern on service behavior lead to low success rate of the matchmaking process. Similarly, the overlooking and unnoticed matchmaking over inequivalent specifications also bring about low qualitative assessments.

\section{Conclusions}

This study offers some insight to the behavioral specification over dynamic and unpredictable environments, in particular, under an incomplete declared intention setting. Two preconditions are asserted to establish a noble model. The first condition is the agent's actions that must be transformed into bipolar attitude scale (favorable-unfavorable). The second is the number of concerning attributes in each domain that must be commonly predefined as a set of finite values, thereby matching process is carried out. The overall results indicate that this proposed model can be used as a deterministic model for eliciting the service execution results of the agent under dynamic and unpredictable environments.

The merit of elaborating a behavioral specification in the form of matrix representation contributes to a deterministic service discovery as "how to access the service" under uncertain environment. This notion is practical enough to be incorporated as a part of the conventional table-based specification approach. Because the matrix representation is closely related to the attribute value fields, each matrix field can be technically transformed to attribute fields. All available matrix manipulation operations help facilitate a practical matchmaking process toward the proposed specification.

Furthermore, the fact that behavioral characteristics of a given service are derived from machine learning process permits parametric attributes to be embedded in service specifications. As such, unknown decision-making situations can be scaled from the specification attributes, whereby principal characteristics of the ordinary specification are preserved, especially for the predominant ones. The proposed matchmaking algorithm also broadens indescribable decision-making situations specified in $\beta$ and $\pi$. The algorithm contributes a significant comparative accuracy of Broker $^{N D}$ and Broker $^{W B}$.

One caveat of the proposed model lies in its scope which is restricted to pairwise matchmaking. It would thus be of interest to further the investigation on classification of service and intention specifications which will in turn enable a more practical matchmaking process.

\section{References}

[1] Quantitative Agent Service Matching, Washington, DC, USA, 2004. IEEE Computer Society.

[2] C. Doulkeridis, E. Valavanis, and M. Vazirgiannis. Towards a context-aware service directory. In Proceedings of the 4th International Workshop on Technologies for E-Services, pages 54-65, September 2003.

[3] B. Fischer, M. Kievernagel, and G. Snelting. Deductionbased software component retrieval. In W. Bibel and P. H. Schmidt, editors, Automated Deduction: A Basis for Applications. Volume III, Applications. Kluwer Academic Publishers, Dordrecht, 1998.

[4] M. Fishbein and A. I. Belief, attitude, intention, and behavior: An introduction to theory and research. Addison-Wesley Publishing, Massachusetts, 1975.

[5] H.-M. Haav and T.-L. Lubi. A survey of concept-based information retrieval tools on the web. In Advances in Databases and Information Systems: Proceedings of 5th East-European Conference, pages 29-41, 2001.

[6] http://www.daml.org/language. DAML+OIL website, 2004.

[7] M. Klein and A. Bernstein. Towards high-precision service retrieval. IEEE Internet Computing, pages 30-36, January 2004.

[8] D. R. Mendoza. Using ontologies in Context-Aware Services Platforms. Master thesis, University of Twente, November 2003.

[9] S. Nakkrasae, P. Sophatsathit, and J. William R.Edwards. Fuzzy subtractive clustering based indexing approach for software components classification. International Journal of Computer \& Information Science, 5(1):63-72, March 2004.

[10] G. G. Richard. Service advertisement and discovery: Enabling universal device cooperation. IEEE Internet Computing, 4(5):18-26, 2000.

[11] A. M. Zaremski and J. M. Wing. Specification matching of software components. ACM Trans. Softw. Eng. Methodol., 6(4):333-369, 1997. 